\title{
Super fast plasma streams as drivers of transient and anomalous magnetospheric dynamics
}

\author{
S. Savin ${ }^{1}$, E. Amata ${ }^{2}$, L. Zelenyi ${ }^{1}$, V. Lutsenko ${ }^{1}$, J. Safrankova ${ }^{3}$, Z. Nemecek ${ }^{3}$, N. Borodkova ${ }^{1}$, J. Buechner ${ }^{4}$, \\ P. W. Daly ${ }^{4}$, E. A. Kronberg ${ }^{4}$, J. Blecki ${ }^{5}$, V. Budaev ${ }^{1,7}$, L. Kozak ${ }^{6}$, A. Skalsky ${ }^{1}$, and L. Lezhen ${ }^{1}$ \\ ${ }^{1}$ IKI, Lab545, Moscow, Russia \\ ${ }^{2}$ IFSI-INAF, Rome, Italy \\ ${ }^{3}$ Charles University, Prague, Czech Republic \\ ${ }^{4}$ Max-Planck Inst. Solar Physics, Katlenburg-Lindau, Germany \\ ${ }^{5}$ Space Research Center, Warsaw, Poland \\ ${ }^{6}$ Kyiv Taras Shevchenko University, Ukraine \\ ${ }^{7}$ NRC Kurchatov Institute, Moscow, Russia
}

Correspondence to: S. Savin (ssavin@iki.rssi.ru)

Received: 1 March 2011 - Revised: 11 November 2011 - Accepted: 1 December 2011 - Published: 3 January 2012

\begin{abstract}
We present multi spacecraft measurements in the magnetosheath (MSH) and in the solar wind (SW) by Interball, Cluster and Polar, demonstrating that coherent structures with magnetosonic Mach number up to 3 - Supermagnetosonic Plasma Streams (SPS) - generate transient and anomalous boundary dynamics, which may cause substantial displacements of the magnetospheric boundaries and the riddling of peripheral boundary layers. In this regard, for the first time, we describe a direct plasma penetration into the flank boundary layers, which is a candidate for being the dominant transport mechanism for disturbed MSH periods.

Typically SPS's have a ram pressure exceeding by several times that of the SW and lead to long-range correlations between processes at the bow shock (BS) and at the magnetopause (MP) on one side and between MSH and MP boundary layers on the other side. We demonstrate that SPS's can be observed both near the BS and near the MP and argue that they are often triggered by hot flow anomalies (HFA), which represent local obstacles to the SW flow and can induce the SPS generation as a means for achieving a local flow balance. Finally, we also discuss other causes of SPS's, both $\mathrm{SW}$-induced and intrinsic to the MSH.

SPS's appear to be universal means for establishing a new equilibrium between flowing plasmas and may also prove to be important for astrophysical and fusion applications.
\end{abstract}

Keywords. Magnetospheric physics (Magnetopause, cusp, and boundary layers; Magnetosheath; Solar windmagnetosphere interactions)

\section{Introduction}

Recent observations by the Cluster, THEMIS and Interball-1 spacecraft (see Savin et al., 2006, 2008, 2010, 2011; Amata et al., 2006, 2011; Hietala et al., 2009; Jacobsen et al., 2009; Zhang et al., 2009, and references therein) indicate the presence of very fast plasma streams inside the MSH. In the spacecraft frame these streams, which we will call SPS's, can last for several tens of seconds and have an extremely high ram pressure. The SPS's, therefore, do not belong to a steady state MSH flow, but are transient periods of enhanced plasma flow. They have been found at all radial distances between the bow shock and the MP with the flow direction often being closer to the original SW flow vector than to the average MSH flow. Apparently such jets have little to do with reconnection as their source, since their kinetic energy density by far exceeds the magnetic energy density. They are thus decoupled from the magnetic dynamics in the MSH and at the MP, although they could trigger secondary driven reconnection of their internal field with the ambient MSH field. It has been suggested that the SW is not the only direct driver of the jets (cf. Nemecek et al., 1998; Savin et al., 2006, 2008, 2011; Amata et al., 2011; Hietala et al., 2009). Flow stratification and turbulence development are rather common phenomena for dissipation of kinetic energy. In this paper we consider processes which have the opposite effect of concentrating plasma flow into narrow jets with high kinetic pressure, even substantially exceeding that of the unshocked SW flow. Its appearance is random, so a kind of chaotization occurs with 


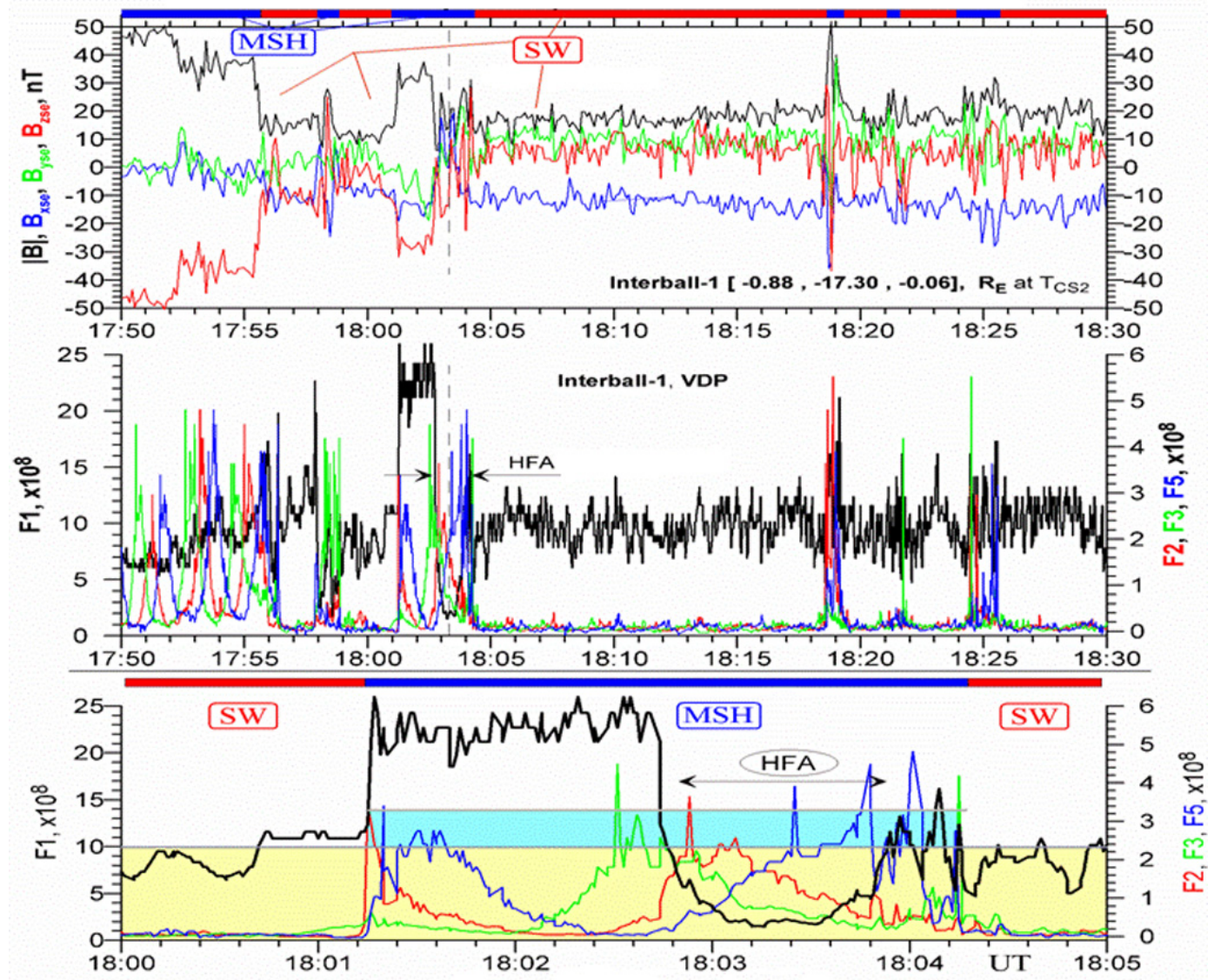

Fig. 1. Interball-1, 5 September 1995, Hot Flow Anomaly (HFA) and SPS observation $\left((-0.9,-17.3,-0.1) R_{\mathrm{E}}\right.$ in GSE at 18:03 UT). Top panel: magnetic field intensity and components in GSE (see color code definition on the left). Middle panel: ion flux from 4 Faraday cups at $1 \mathrm{~Hz}$ sampling in $\mathrm{cm}^{-2} \mathrm{~s}^{-1}$. F1 from the Sun direction, F2, F3 and F5 (see color code definition on the right) transverse to the Sun-Earth line, rotating around this line with a 2 min period. Bottom panel: blow-up of the middle panel around the HFA; yellow (cyan) shading shows the average sum of fluxes from the 4 Faraday cups in the SW (in the HFA and SPS).

quantum-like objects imbedded - the jets. SPS's represent a kind of extreme events, which drive an intermittent turbulence in magnetopause and MSH turbulent boundary layers (Savin et al., 2011). The detailed mechanism of the jet generation is yet to be understood, as is the case for the accretion in quasars and stars, water drop impacts, TOKAMAK boundary layers etc. (Savin et al., 2008). Here we show that local obstacles to the flow such as HFA's can trigger SPS's just due to the local conservation of flux. This finding supports the phenomenological model of Savin et al. (2011) and is not in contradiction with the model of Hietala et al. (2009).

\section{Data description}

Figure 1 displays Interball-1 magnetic field and plasma data from 17:50 to 18:30 UT on 5 September 1995. The top panel shows the magnetic field module and vector (in GSE, Geocentric Solar Ecliptic, coordinates). In the middle panel ion fluxes are plotted from four Faraday cups: F1, black line, shows anti-sunward flux; F2, F3 and F5 show fluxes from 3 perpendicular directions, all perpendicular to the Sun-Earth line. Measurements in the MSH and in the SW, near the BS subsolar point, are highlighted at the top through colored bars. The MSH can be identified from large $|B|$ values and from the appearance of perpendicular flows behind the BS (see colored lines in the middle panel, modulated at the satellite $2 \mathrm{~min}$ spin period). The bottom panel is a blow-up of the middle one from 18:00 to 18:05 UT. The most prominent HFA, which is indicated in the middle panel by two arrows, represents a substantial (i.e. more then 50\%) local drop of the antisunward flux, which can be seen in the middle panel at about 17:56, 17:58 and 18:03 UT. On average, during the 16:30-17:30 UT quiet time interval, the ratio between the ion fluxes measured in the MSH by Interball- 1 and in the SW by Geotail (not shown) is $\sim 1.5$ (i.e. close to the average ratio for the Interball-1 BS crossings, cf. Savin et al., 2011). For the extended HFA interval 17:59-18:10 UT one gets that same ratio, similar to the SW average level at 17:59-18:01 and 
18:05-18:10 UT at Interball-1. Taking into account that the SW Wind data (time-lagged from L1) agree with the Geotail data (not shown), one can conclude that the average flux around the HFA in the bottom panel of Fig. 1 is conserved (cf. Savin et al., 2011). The gain of "1.5" in the flux is mostly due to the plasma density rise at the BS at the HFA leading edge (cf. Hietala et al., 2009). The BS inbound/outbound crossings at 18:01:10-18:04:10 UT are evident from the appearance of perpendicular ion fluxes (see the colored curves in the bottom panel of Fig. 1). We call "SPS" (cf. Savin et al., 2011) the highly enhanced flow at the leading front of the HFA at 18:01:10-18:02:40 UT: its value exceeds by about 2.5 times that of the quiet MSH at 17:50-17:55 UT (middle panel of Fig. 1); its angle is only $\sim 7^{\circ}$ to the $-\mathrm{X}$ GSE axis (while it is $24^{\circ}$ in the quiet MSH); this SPS compensates the flux drop in the following HFA, letting the average flux in the HFA vicinity to be conserved. Unfortunately, no ion speed data are available to calculate the ram pressure for Fig. 1.

If one looks at the shorter HFA's at 17:55 and 17:58 UT, one sees SPS's bounding one or two HFA sides (here the flux balance cannot be checked due to the short HFA duration). Similar cases seen by Interball-1, Geotail and THEMIS (Savin et al., 2011) demonstrate that the local flux balance should not be satisfied only along an orbit, which is close to the HFA nose where the flow stagnation and bifurcation take place. Note also the SPS at 18:19 UT: no HFA is seen, while the case can be a periphery crossing of an SPS/ HFA. As regards SW current sheets, which are believed to trigger the HFA's (Sibeck et al., 1998; Schwartz et al., 2000), one can see them in the top panel of Fig. 1, while the BS crossings is masking the picture. We have checked with Geotail, Wind and IMP-8 data in the SW that a clear current sheet corresponds only to the last HFA (bottom panel of Fig. 1), while the two leading HFA's correlate with impulsive magnetic fluctuations with a complicated structure.

Figure 2 displays the GSE orbit and ion velocity vectors during an outbound path of the Cluster spacecraft at $\sim 600 \mathrm{~km}$ separation on 17 March 2001, traversing the MSH from the MP to the BS. The top panel gives the magnetosonic Mach number $\left(M_{\mathrm{MS}}\right)$ with vertical dashed lines corresponding to the SPS's described in Amata et al. (2011). We will concentrate on the 3 most prominent SPS's, marked by "A, $\mathrm{B}, \mathrm{H}$ ". The velocity vectors for these SPS's are displayed in Inset 1 of Fig. 2. The background MSH flow velocities are marked by " $b$ " in the top left part, while the blue dashed line depicts a plasma streamline and the thick grey line shows the Cluster orbit. Throughout this event the GSM $B_{\mathrm{Z}}$ component was positive both in the MSH and in the SW, thus excluding dayside subsolar-point reconnection. In our case the kinetic energy density $W_{\mathrm{k}} \gg W_{\mathrm{b}}$ (magnetic pressure) within the jets, that also excludes reconnection as a local source for the MSH jets (Amata et al., 2011). Hence the B-jet could have a similar jet in the MSH far away from the MP as its source (cf. $\mathrm{H}$-jet). The angle between the $\mathrm{H}$-jet and the local magnetic field on Cluster 1 was $\sim 97^{\circ}$ suggesting cross-field accelera-
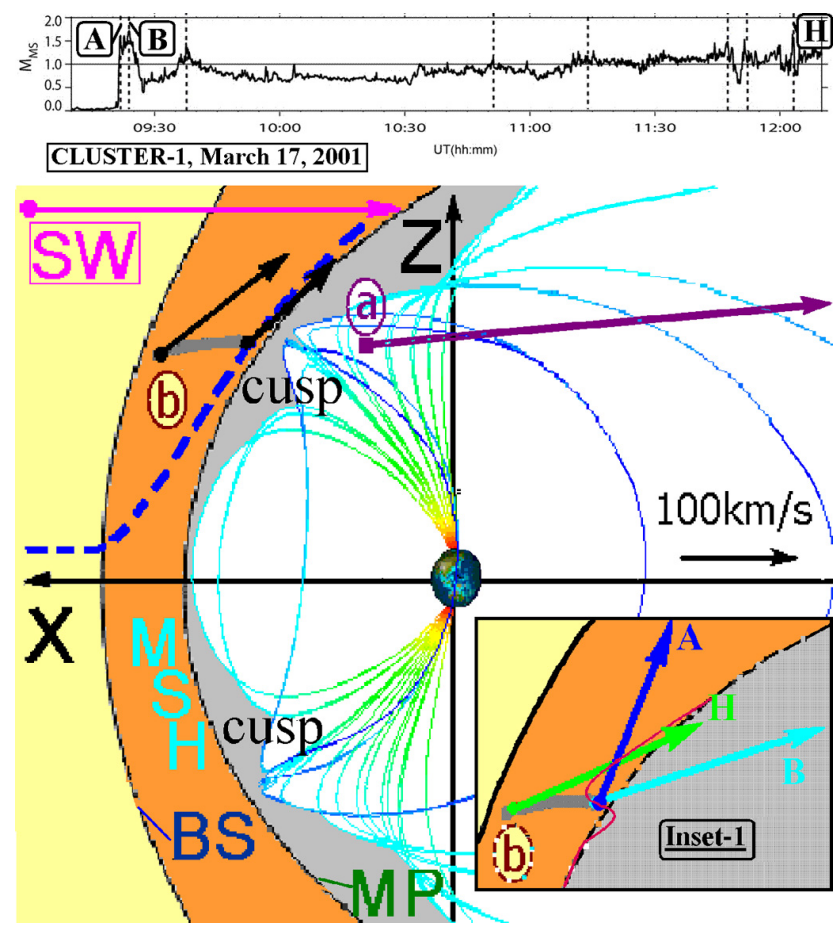

Fig. 2. Top panel: plot of the Cluster 1 magnetosonic Mach number $M_{\text {MS }}$ for a MSH traversal on 17 March 2001 (see details in text and in Amata et al., 2011). Bottom panel: scheme of the SPS interaction with the magnetosphere (GSE frame, all for 17 March 2001 except the arrow marked by circled "a"). From left to right: SW, BS, MSH, MP and magnetic field lines in the magnetosphere calculated through OVT (http://ovt.irfu.se) using the SW dynamic pressure and IMF at 11:00 UT on 17 March 2001. The background flow velocities in the MSH are marked by "b" and black arrows in the top left part; the blue dashed line shows a plasma streamline; the thick grey line is the Cluster orbit; the violet arrow marked "SW" shows the average SW velocity. The dark violet arrow marked "a" displays the velocity vector for the SPS at 06:33 UT on 7 February 2001 (Cluster 1), marked by a grey arrow in Fig. 3. Inset 1 repeats a portion of the main figure: instead of quiet MSH velocities, streamline and magnetic field lines, the inset shows velocity vectors for the "A", "B" and "H" SPS's and the distorted MP (see the thin red line) from Amata et al. (2011).

tion for a local source (as suggested by Savin et al., 2011), while that of the B-jet is $\sim 137^{\circ}$, probably due to the background magnetic field piling-up. The $W_{\mathrm{k}}$ value in the $\mathrm{H}$-jet is close to that of the B-jet, while the profile is narrower. As one can see from Savin et al. (2008), such jets deflected towards $-X$ GSE, constitute a minor portion of the jets, most of which are close to the MSH flow direction (cf. the black arrow near the MP). But even as a minor portion, the skewing jets can play a definitive role for the flank MP formation and transport processes.

In support of this statement, in Fig. 2 the dark-violet arrow (marked by the circled "a") shows another Cluster jet near the MP on 7 February 2001 at $\sim 15: 00$ MLT. Its $W_{\mathrm{k}}$ is 


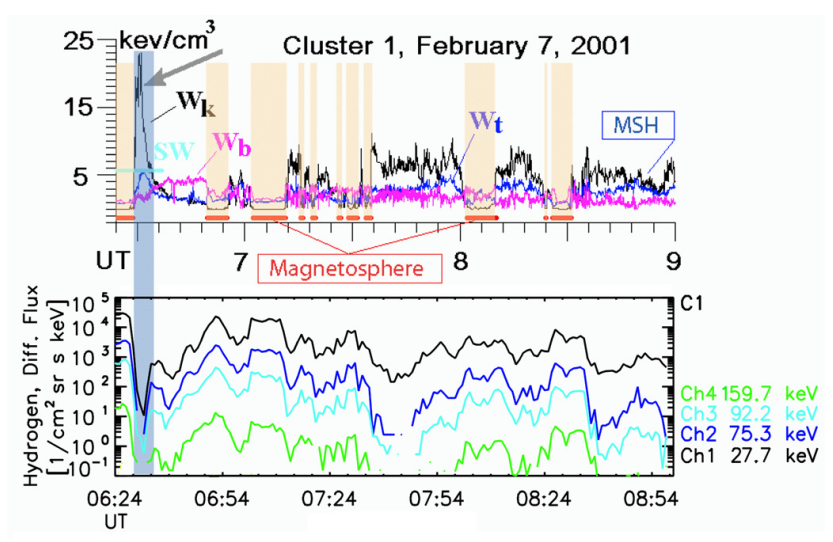

Fig. 3. 7 February 2001. Top panel: Cluster 1 ram $W_{\mathrm{k}}$ (black), ion thermal $W_{\mathrm{t}}$ (blue) and magnetic $W_{\mathrm{b}}$ (violet) pressure; WIND ram pressure in SW during the first jet (outlined by gray arrow, see also Fig. 2) is shown by magenta horizontal line marked "SW". Red thick lines above the horizontal axis and vertical pink shadings highlight the magnetosphere; the quiet MSH is indicated on the right by the framed "MSH". Bottom panel: Proton intensities observed by Cluster 1, RAPID. The vertical blue transparent bar highlights the time period when the high-energy-density "super-jet" occurred simultaneously with the proton intensity deepest drop.

extremely strong (see the grey arrow in Fig. 3, where the simultaneous $W_{\mathrm{k}}$ level in the $\mathrm{SW}$ is given by the horizontal magenta line), being almost 4 times larger than the SW $W_{\mathrm{k}}$, its magnetosonic Mach number is $M_{\mathrm{MS}} \sim 2$ (in the quiet MSH, marked by framed "MSH", $M_{\mathrm{MS}} \leq 1$ ) and its direction forms an angle of about $10^{\circ}$ with the $-\bar{X}$ GSE axis. This jet is the innermost one followed by $\sim 2 \mathrm{~h}$ of crossing of the MP boundary layer, riddled by weaker jets, similar to the B-jet of Fig. 2. Data pertaining to the magnetosphere are highlighted in Fig. 3 by vertical colored bars corresponding to dominant magnetic pressure ( $W_{\mathrm{b}}$, violet line), which is falling with time and is negligible relative to the $W_{\mathrm{k}}$-spikes in the MSH. It means that the SPS passed the maximum magnetospheric magnetic field over the cusp (cf. Fig. 2). RAPID data (Wilken et al., 2001) confirm the magnetosphere encounters by Cluster-1, displaying the trapped energetic protons inside the magnetosphere. Outside the MP the leaking protons are seen, excluding the "super-jet" (marked with vertical dark shading and gray arrow) with the deepest minimum in flux at the lowest energy. Surprisingly, the latter indicate the absence of local acceleration of energetic particles by the high energy-density "super-jet". Instead, the energetic particle depletion infers that the magnetic field inside the "super-jet" is not reconnected with the magnetospheric magnetic field. The depletion looks rather strange also in view of the anticipated thin jet boundaries, the width of which should be comparable with the thermal proton gyroradius at the leading edge of the jet (Savin et al., 2006, 2010). In this case gyro-diffusion should take place (Kuznetsov et al., 2007). Such a minimum could occur if the jet were skewing the closed magneto- spheric field lines faster than the proton gyroperiod $(\sim 30 \mathrm{~s})$. This consideration yields an upper limit of $\sim 10000 \mathrm{~km}$ for distance along the jet, when the jet was inside the magnetosphere; this looks to be realistic (cf. Fig. 2), but cannot be verified from the cross-Cluster data, as the Cluster separation is only $600 \mathrm{~km}$.

The flow in the disturbed MSH is supersonic, as the thermal energy density (blue line) $W_{\mathrm{t}}<W_{\mathrm{k}}$, while in the quiet MSH $W_{\mathrm{t}} \sim W_{\mathrm{k}}>W_{\mathrm{b}}$. The most powerful spikes, SPS's, are supermagnetosonic $\left(W_{\mathrm{t}}+W_{\mathrm{b}}<W_{\mathrm{k}}\right)$. Hence in the case of Fig. 3 the SPS's can be the general factor for the transport of solar wind plasma into the mantle at the geotail boundary. Numerically, the plasma flow in the boundary layer at 06:25-07:25 UT is carried mostly by the skewing SPS's, so that it constitutes $2 / 3$ of that in the SW from WIND data. At the same time, both modeling and experimental studies highlight $10 \%$ as a characteristic value for the MSH flow penetration into high-latitude boundary layer through diffusive-like or reconnection-like processes (see e.g. Savin et al., 2005a, $\mathrm{b}$, and references therein).

Coming back to the B-jet, it is closer to the SW direction than the average MSH flow (see the black arrow near the MP at the top left side of Fig. 2) and it most probably governs the main MP indentation (see the red curve in Inset 1). While the weaker A-jet closest to the MP is almost elongated to the average MP (black dashed line in Inset 1), the local MP normal forms a $97^{\circ}$ angle with the average one. So, the A- and B-jets result in the MP distortion up to several $R_{\mathrm{E}}$ (cf. Inset 1 and Amata et al., 2011). For instance, in Jacobsen et al. (2009) the boundary deformations, comparable with the MSH width, are also shown, and Savin et al. (2011) demonstrated that SPS's deform the MP, approaching a pressure balance at the position of the innermost THEMIS-E spacecraft.

Let us go back to the comparison of Figs. 2 and 3: the "super-jet" at 06:33 UT on 7 February 2001 is clearly behind the polar cusp. This demonstrates that SPS's similar to that of Figs. 1-3 can riddle the flank magnetosphere and flow freely tailward in the mantle or plasma sheet boundary layer. No prominent correlation with the $B_{\mathrm{Z}} \mathrm{IMF}$ has been found, so reconnection can play a minor/secondary role in the process under study (cf. Savin et al., 2008).

In a different case on 23 April 1998 (Fig. 4), at the moment of the SW flux fall by $\sim 3$ times at 23:00 UT, both Interball1 and Polar (being $\sim 4 R_{\mathrm{E}}$ apart) registered tremendous jets being absent in the SW Wind data. At 21:45-23:00 UT Polar was outside the MP in the northern cusp throat, where the MHS flux is depressed and even stagnant (see top panel of Fig. 4). Another SPS spike at 22:00 UT (visible also at Interball-1, see bottom panel) corresponds to a narrow drop in the SW flux (see thick green line in the bottom panel), one more less prominent spike correlates with a SW flux jump. It is an indication that SPS's could regularly accompany SW pressure jumps (cf. Savin et al., 2011). At about 22:1522:45 UT Interball-1 also crossed the cusp throat with stagnant plasma gradually approaching the free MSH flow (see 


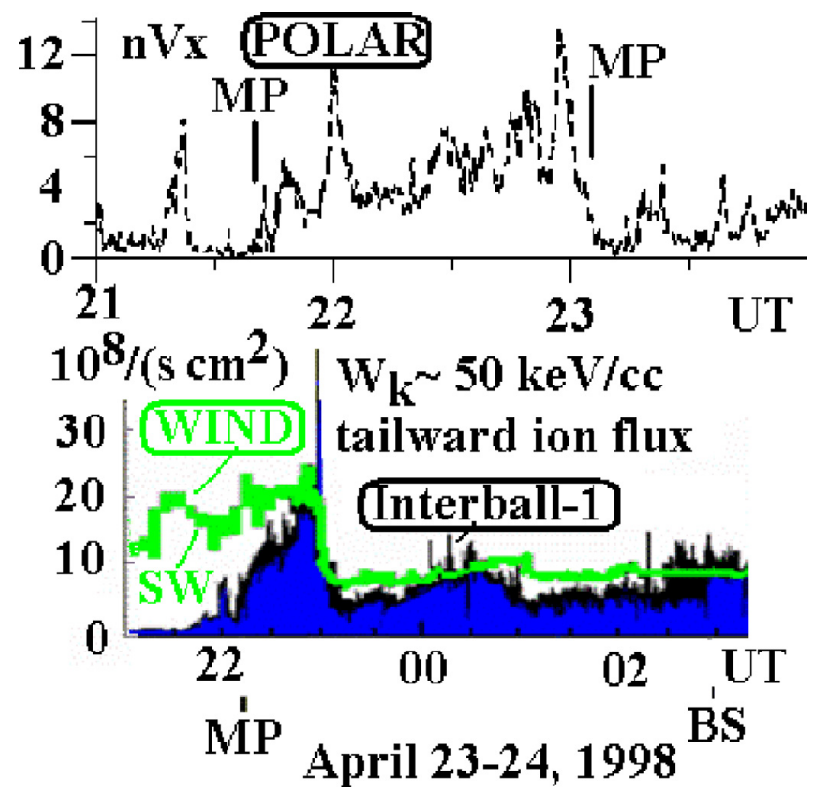

Fig. 4. 23 April 1998. Top panel: antisunward ion flux $n V_{\mathrm{x}}$ in $\left.10^{8} \mathrm{~s}^{-1} \mathrm{~cm}^{-2}\right)$ as seen by Polar at $\left.(6,-1,4) R_{\mathrm{E}} \mathrm{GSM}\right)$. Bottom panel: antisunward ion flux $n V_{\mathrm{X}}$ in $\left(10^{8} \mathrm{~s}^{-1} \mathrm{~cm}^{-2}\right)$ as seen by Interball-1 at $(2,-4,8) R_{\mathrm{E}}$ GSM (blue shadowed black line) and by WIND (thick green line, time-lagged from L1 to Interball-1).

the shadowed curve in the bottom panel of Fig. 4), which was comparable with the plasma flux in the SW (green thick line). The role of the SPS at $\sim 23: 00 \mathrm{UT}$ is qualitatively clear: the jet serves to remove the plasma "excess" in the MSH, which should become much less dense in response to the SW dynamic pressure drop. A problem is: why is the flow rising in the SPS transient spike, instead of gradual falling? And why is the SPS duration rather standard, resembling a phonon/soliton behavior? Surprisingly, a Geotail SPS in the similar conditions on 16 April 1996, almost repeated those of Fig. 4 (see Savin et al., 2011). A difference is that in the Geotail case a clear HFA precedes the SPS, the HFA has been also seen by Interball-1 $\sim 15 R_{\mathrm{E}}$ in front of Geotail. After the "super-jet", the MSH flux, $n V_{\mathrm{x}}$, (Interball-1, see bottom panel in Fig. 4) drops even below that of the SW (green line), then gradually approaches it. After 3 SPS's at $\sim 00: 20$ UT (black spikes) the transient process repeats: the MSH $n V_{\mathrm{x}}$ drops again until the SPS and BS multiple crossings at 02:00-03:00 UT. Such a low $n V_{\mathrm{x}}$ in the outer MSH relative to that of SW is not typical for the Interball-1 orbit. Probably, the SPS's move fast past Interball-1 in their transverse direction, giving much larger input to the total flux balance between MP and BS as compared with time-averaging along the orbit (cf. Savin et al., 2006, 2008, 2011). In any case, the SPS input to the flux balance should be rather substantial ( $\sim 30 \%$, see Savin et al., 2008, 2011, and references therein).

\section{Discussion and conclusions}

As shown in Fig. 1, SPS's are bounding HFA's and can keep the local flux balance, being, most probably, dense and supermagnetosonic in the MSH because of the BS rotation in interaction with a local obstacle (cf. Hietala et al., 2009; Savin et al., 2011). However, as mentioned above, HFA's and SW current sheets are not the only sources of SPS's (Nemecek et al., 1998; Savin et al., 2006, 2008, 2010; Amata et al., 2011; Hietala et al., 2009). Savin et al. (2008, 2011) outline that $\mathrm{MSH}$ and boundary resonances at a few $\mathrm{mHz}$ can substantially affect the SPS appearance and boundary dynamics. Zastenker (2004) and Shue et al. (2009) demonstrate the appearance of SPS-like flow spikes without SW disturbances for stable radial IMF: in such cases, non-linear foreshock disturbances, most probably, represent local obstacles for the SW flux and trigger SPS's similarly to the HFA case (cf. Hietala et al., 2009). Figure 4 and Savin et al. (2011) depict "superjets" as the MSH transient reaction to SW flux drops. In the case of Savin et al. (2011), Interball-1 registered an HFA triggered by a SW pressure drop, while in other published cases the SW magnetic discontinuities were believed to trigger the HFA's. From the discussion above and from many other experimental data, we suggest that SPS's are universal means for approaching a new equilibrium through transient processes, including eigen-oscillations of the MSH and magnetospheric boundaries.

As for the previous suggestions for the SPS generation mechanisms, sonic (at maximum) jets could be generated in the MSH either by flow self-focusing, or by surface charge at moving magnetospheric boundaries (see Savin et al., 2006, 2008, 2011, and references therein). The Laval-nozzle effect was believed to accelerate the "super-jets" (cf. Fig. 3, gray arrow) to supermagnetosonic speeds as they passed through the maximum of magnetic field strength over the cusp (cf. Fig. 3, see Savin et al., 2005a, and references therein). However, the regular occurrence of SPS's in the dayside MSH (see Figs. 13 and Savin et al., 2008, 2011, and references therein) yields that the near bow shock region is the dominant place for generation of fast SPS's. Hietala et al. (2009) highlight an evident way for the penetration of supermagnetosonic jets and dissipation in the MSH. But their paper alone, being an obligatory step in the SPS nature understanding, does not completely explain the SPS mechanism: Savin et al. (2011) demonstrate, using both Interball-1 and THEMIS data, the SPS appearance first in the SW (which does not care of the downstream BS orientation) either just ahead of an HFA or in conjunction with jumps of SW dynamic pressure (to be further investigated, cf. Fig. 4). The plasma acceleration around the flow anomalies (the HFA being a particular type of flow anomaly) is proposed to occur due to a polarization electric field at the flanks of the anomalies (Savin et al., 2011): the fact that in Fig. 2 of this paper the magnetic field is almost transverse to the $\mathrm{H}$ - jet velocity in the BS vicinity, confirms this mechanism. 
Table 1. Scaling $\eta$ for Fig. 5 (see also Savin et al., 2011).

\begin{tabular}{clc}
\hline No. in Fig. 5 & & $\eta$ \\
\hline 2 & TBL, $B_{\mathrm{Z}}$ (CLUSTER, 2 Feb 2003, <2 Hz, see Kuznetsov et al., 2007) & 2.2 \\
3 & TBL, $n V_{\mathrm{z}}$ (CLUSTER, 2 Feb 2003, <2 Hz, see Kuznetsov et al., 2007) & 1.94 \\
4 & TBL, $B_{\mathrm{y}}$, (GEOTAIL, 16 Apr 1996, 18:54-20:04 UT, see Savin et al., 2011) & 2 \\
6 & TBL, $n V$ (GEOTAIL, 16 Apr 1996, 18:54-20:04 UT, see Savin et al., 2011) & 1.3 \\
\hline
\end{tabular}

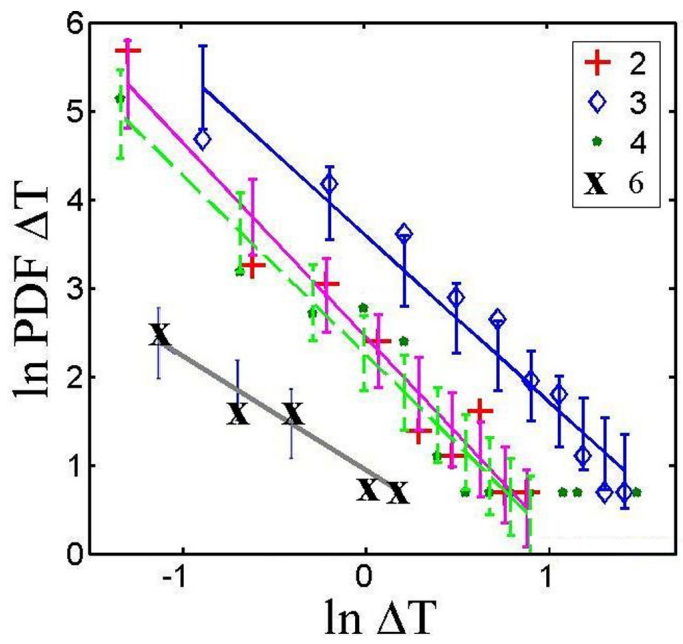

Fig. 5. The distribution functions of waiting times for extreme events (peaks above 2 standard deviations) in logarithmic scale. Numbers in the upper right corner correspond to Table 1. The data are approximated by $P(t) \sim t^{\eta}$ (the "scaling" corresponds to the straight lines - see details in Savin et al., 2011).

Our conclusive hypothesis to be further verified, is that SPS's represent the eigen-modes for space-limited open systems with moving matter flows in a variety of media. Their quasi- quantum signatures are waiting for the proper theoretical background (see Savin et al., 2005, 2008, 2011, and references therein). Namely those coherent structures enable to provide the long- range correlations being responsible for intermittency and multi-fractality in a statistical sense. The dependence of $\zeta(q) / \zeta$ (3) from $q$ in Savin et al. (2011) (see also references therein) differs from the known cascade models of turbulence with intermittency (e.g. She - Leveque), demonstrating the existence of multi-fractal properties and of generalized self-similarity. These properties are associated with "long-range" correlations of global and local scales. In our case, the long-range correlations, most probably, are due to the SPS coherent structures. To confirm this, we looked for extreme events in the data, i.e. for amplitude bursts greater than 2 standard deviations. Actually, Savin et al. (2008, 2011) made use of this definition in order to identify jets in the case of a plasma flux or kinetic energy density. Next, we constructed the distribution functions of waiting times, $\Delta T$, between successive extreme events (see Fig. 5). If extreme events occur as a result of a trivial random process, the distribution function of waiting times $P(\Delta T) \sim \Delta T^{\eta}$ should have the scaling $\eta \sim-1$ (see Budaev et al., 2006). However, Fig. 5 and Table 1 show a systematic deviation of $\eta$ from "-1" (see details in Savin et al., 2011). This confirms that the extreme events in terms of kinetic energy concentration in plasma streams, i.e. SPS's, are the carriers of "long-range" correlations in the statistical sense. In order to conduct an additional test of the SPS role in establishing long-range correlations, we randomized the phases of $n V_{\mathrm{Z}}$ (the direction " $Z$ " in this case, in the limit of $20^{\circ}$, coincides with the average normal to the magnetopause) for the 2 February 2003 event (see Kuznetsov et al., 2007, No. 3 in Table 1 and Fig. 5). As expected, the slope of the distribution function of the extreme events became close to " -1 ". The structure functions approached that of the Kolmogorov K41 model. At the same time, more than $85 \%$ of the extreme events/peaks, in the plasma flow with amplitude of more than 2 standard deviations of the original signal, disappeared. In other words, the long-range correlations disappeared together with the extreme events, i.e. SPS's (cf. Savin et al., 2011).

The qualitative SPS effect for the plasma and momentum transport across magnetic boundaries is obvious from Figs. 2 and 3: the MP can be riddled by the tremendously concentrated jets providing a way for the dominant transport inward/through the periphery magnetosphere during disturbed MSH periods (cf. the flow in Fig. 5 in the boundary layer at 06:25-07:25 UT, estimated above as being $\sim 6$ time larger than that of typical penetrating flow due to diffusion or reconnection). Another general point is that SPS's transfer middle-scales ( $\sim$ SPS transverse width) and microscales ( $\sim$ the scales of SPS boundaries and substructures) at the maximum - supermagnetosonic - speed throughout the SWmagnetosphere system, generally because of their flow (and energy) density concentration.

Finally, we assert that the flow structuring/jetting is a fundamental plasma feature to be studied in details, having at least an importance comparable with that of magnetic reconnection. The jets occur to be detected in the near-Earth space since late sixties. Understanding their dynamic properties and origin could essentially modify the approach to SWmagnetosphere interaction and should also shed light on heliospheric, laboratory and astrophysical plasma interactions.

Acknowledgements. The work was supported by RAS Programs OFN15 and P-4 and grants MNiSW N N307 101935, RFFR 
100200135, 10-02-01063 10-02-93114 and 11-02-90491, grant of Leading Scientific Schools NSc I-3200.2010.2, and DRRD-RFFR F no. 40.2/053. We thank K. W. Ogilvie and the SWE team for providing WIND solar wind dynamic pressure data, S. Fuselier for Polar TIMAS data, G. Zastenker for Interball VDP ion flux data. P. Daly and E. Kronberg thank the Deutsches Zentrum für Luft und Raumfahrt (DLR) for supporting the RAPID instrument at MPS under grant 50 OC 1101.

Guest Editor M. Balikhin thanks two anonymous referees for their help in evaluating this paper.

\section{References}

Amata, E., Savin, S., André, M., Dunlop, M., Khotyaintsev, Y., Marcucci, M. F., Fazakerley, A., Bogdanova, Y. V., Décréau, P. M. E., Rauch, J. L., Trotignon, J. G., Skalsky, A., Romanov, S., Buechner, J., Blecki, J., and Rème, H.: Experimental study of nonlinear interaction of plasma flow with charged thin current sheets: 1 . Boundary structure and motion, Nonlin. Processes Geophys., 13, 365-376, doi:10.5194/npg-13-365-2006, 2006.

Amata, E., Savin, S. P., Ambrosino, D., Bogdanova, Y. V., Marcucci, M. F., Romanov, S., and Skalsky, A.: High kinetic energy density jets in the Earth's magnetosheath: A case study, Planet. Space Sci., 59, 482-494, doi:10.1016/j.pss.2010.07.021, 2011.

Budaev, V. P., Takamura, S., Ohno, N., and Masuzaki, S.: Superdiffusion and multifractal statistics of edge plasma turbulence in fusion devices, Nuclear Fusion, 46, S181, 2006.

Hietala, H., Laitinen, T. V., Andréeová, K., Vainio, R., Vaivads, A., Palmroth, M., Pulkkinen, T. I., Koskinen, H. E. J., Lucek, E. A., and Rème, H.: Supermagnetosonic Jets behind a Collisionless Quasiparallel Shock, Phys. Rev. Lett., 103, 245001-1-245001-4, 2009.

Jacobsen, K. S., Phan, T. D., Eastwood, J. P., Sibeck, D. G., Moen, J. I., Angelopoulos, V., McFadden, J. P., Engebretson, M. J., Provan, G., Larson, D., and Fornacon, K.H.: THEMIS observations of extreme magnetopause motion caused by a hot flow anomaly, J. Geophys. Res., 114, A08210, doi:10.1029/2008JA013873, 2009.

Kuznetsov, E. A., Savin, S., Amata, E., Dunlop, M., Zelenyi, L. M., Panov, E. V., Büchner, J., Romanov S. A., Blecki, J., Rauch, J. L., and Nikutowski, B.: Strong Space Plasma Magnetic Barriers and Alfvénic Collapse, JETP Lett. 85, 236-241, 2007.

Němeček, Z., Šafránková, J., Pech, L., Sibeck, D. G., Kokubun, S., Mukai, T.: Transient flux enhancements in the magnetosheath, Geophys., Res. Lett., 25, 1273-1276, 1998.

Savin, S., Skalsky, A., Zelenyi, L., Avanov, L., Borodkova, N., Klimov, S., Lutsenko, V., Panov, E., Romanov, S., Smirnov, V., Yermolaev, Yu., Song, P., Amata, E., Consolini, G., Fritz, T. A., Buechner, J., Nikutowski, B., Blecki, J., Farrugia, C., Maynard, N., Pickett, J., Sauvaud, J. A., Rauch, J. L., Trotignon, J. G., Khotyaintsev, Y., and Stasiewicz, K.: Magnetosheath interaction with the high latitude magnetopause, Surv. Geophys., 26, 95133, doi:10.1007/s10712-005-1874-4,2005a.

Savin, S., Zelenyi, L., Amata, E., Büchner, J., Blecki, J., Greco, A., Klimov S., Lopez, R.E., Nikutowski, B., Panov, E,. Pickett, J., Rauch, J.L., Romanov, S., Song, P., Skalsky, A., Smirnov, V., Taktakishvili, A., Veltri, P., and Zimbardo, G.: Magnetosheath interaction with high latitude magnetopause: dynamic flow chaotization, Planet. Space Sci., 53, 133-140, 2005 b.
Savin, S., Amata, E., Andre, M., Dunlop, M., Khotyaintsev, Y., Decreau, P. M. E., Rauch, J. L., Trotignon, J. G., Buechner, J., Nikutowski, B., Blecki, J., Skalsky, A., Romanov, S., Zelenyi, L., Buckley, A. M., Carozzi, T. D., Gough, M. P., Song, P., Reme, H., Volosevich, A., Alleyne, H., and Panov, E.: Experimental study of nonlinear interaction of plasma flow with charged thin current sheets: 2. Hall dynamics, mass and momentum transfer, Nonlin. Processes Geophys., 13, 377-392, doi:10.5194/npg-13377-2006, 2006.

Savin, S., Amata, E., Zelenyi, L., Budaev, V., Consolini, G., Treumann, R., Lucek, E., Šafránková, J., Nmeek, Z., Khotyaintsev, Y., André, M., Büchner, J., Alleyne, H., Song, P., Blecki, J., Rauch, J. L., Romanov, S., Klimov, S., and Skalsky. A.: High kinetic energy jets in the Earth's magnetosheath: Implications for plasma dynamics and anomalous transport, JETP Letters, 87, 593-599, 2008.

Savin, S., Zelenyi, L., Amata, E., Budaev, V., Büchner, J., Blecki J., Balikhin, M., Klimov, S., Korepanov, V. E., Kozak, L., Kudryashov, V., Kunitsyn, V., Lezhen, L., Milovanov, A. V., Nĕmeček, Z., Nesterov, I., Novikov, D., Panov, E., Rauch, J. L., Rothkaehl, H., Romanov, S., Šafránková, J., Skalsky, A., and Veselov, M.: ROY - A multiscale magnetospheric mission, Planet. Space Sci., 59, 7 doi:10.1016/j.pss.2010.05.001, 2010.

Savin S., Budaev, V., Amata, E., Zelenyi, L., Sibeck, D., Lutsenko, V., Borodkova, N., Zhang, H., Angelopoulos, V., Šafránková, J., Němeček, Z., Blecki, J., Büchner, J., Kozak, L., Romanov, S., Skalsky, A., and Krasnoselsky, V.: Dynamic interaction of plasma flow with boundary layer of geomagnetic trap, JETP Letters, 93, 827-846, 2011.

Schwartz, S. J., Paschmann, G., Sckopke, N., Bauer, T. M., Dunlop, M., Fazakerley, N., and Thomsen, M. F.: Conditions for formation of hot flow anomalies at Earth's bow shock, J. Geophys.Res., 105, 12639-12650, 2000.

Shue, J.-H., Chao, J.-K., Song, P., McFadden, J. P., Suvorova, A., Angelopoulos, V., Glassmeier, K. H., and Plaschke, F.: Anomalous magnetosheath flows and distorted subsolar magnetopause for radial interplanetary magnetic fields, Geophys. Rev. Lett., 36, L18112, doi:10.1029/2009GL039842, 2009.

Sibeck, D., Borodkova, N., Zastenker, G., Romanov, S., and Sauvaud, J.: Gross deformation of the dayside magnetopause, Geophys. Res. Lett., 25, 453-456, 1998.

Wilken, B., Daly, P. W., Mall, U., Aarsnes, K., Baker, D. N., Belian, R. D., Blake, J. B., Borg, H., Bchner, J., Carter, M., Fennell, J. F., Friedel, R., Fritz, T. A., Gliem, F., Grande, M., Kecskemety, K., Kettmann, G., Korth, A., Livi, S., McKenna-Lawlor, S., Mursula, K., Nikutowski, B., Perry, C. H., Pu, Z. Y., Roeder, J., Reeves, G. D., Sarris, E. T., Sandahl, I., Sraas, F., Woch, J., and Zong, Q.-G.: First results from the RAPID imaging energetic particle spectrometer on board Cluster, Ann. Geophys., 19, 1355-1366, doi:10.5194/angeo-19-1355-2001, 2001.

Zastenker, G.: An overview of new concepts deduced from INTERBALL solar wind investigations, in: Multiscale processes in the Earths magnetosphere: from INTERBALL to CLUSTER, NATO Science Series, II, 178, 37-56, 2004.

Zhang, H., Zong, Q.-G., Sibeck, D. G., Fritz, T. A., and McFadden, J. P.: Dynamic motion of the bow shock and the magnetopause observed by THEMIS spacecraft, J. Geophys. Res., 114, A00C12, doi:10.1029/2008JA013488, 2009. 